\title{
Definición del nivel de interferencias en la transmisión de señales digitales de radiodifusión en modo híbrido
}

\section{Transmission Interferences Level Measurement for Digital Audiobroadcasting Signals Transmitted on Hybrid Mode}

\author{
Moumtadi F. \\ Departamento de Electrónica \\ Facultad de Ingeniería \\ Universidad Nacional Autónoma de México \\ Correo:fatimoum@hotmail.com \\ Delgado-Hernández J.C. \\ Escuela de Ingeniería \\ Universidad Anáhuac del Norte \\ Correo:cardelher@gmail.com
}

\author{
Rodríguez-Huerta J. \\ Departamento de Telecomunicaciones \\ Facultad de Ingeniería \\ Universidad Nacional Autónoma de México \\ Correo:ar_zimraphel@hotmail.com
}

Información del artículo: recibido: julio de 2010, aceptado: septiembre de 2011

\section{Resumen}

Una de las estrategias más importantes para impulsar la introducción de la radiodifusión digital en estaciones comerciales se basa en el uso de la actual infraestructura de transmisión analógica para enviar señales tanto analógicas como digitales a través de un mismo medio y rango de frecuencias, utilizando un adecuado esquema de modulación. Este modo de transmisión, denominado "modo híbrido", se basa en el uso de las frecuencias de guarda entre frecuencias activas de transmisión analógica para el envío de señales digitales. Sin embargo, cuando las estaciones transmisoras utilizan frecuencias adyacentes con guardas de protección mínimas entre ellas, la información digital puede estar sujeta a una fuerte interferencia. En este trabajo se analiza el comportamiento de los estándares AM In Band-On channel (AM IBOC) y Digital Radio Mondiale (DRM) transmitiendo en modo híbrido desde frecuencias adyacentes, se realiza una simulación para calcular el nivel de interferencia y se detallan las implicaciones del fenómeno y sus posibles soluciones, tomando como ejemplo el caso de tres estaciones comerciales de la Ciudad de México.

\section{Descriptores}

- señales digitales

- DRM

- IBOC

- modo de transmisión híbrido 


\begin{abstract}
One of the most important strategies developed to boost the commercial introduction of new digital radiobroadcast standards is based on the use of the present AM transmission infrastructure to send both analog and digital signals over the same medium and range of frequencies through a suitable scheme of modulation. This mode of transmission is denominated "hybrid mode", and is based on the use of the keeper range of frequencies between carriers of analogical transmission to send the digital signals. However, radiobroadcast over adjacent frequencies with minimal guard intervals between them could generate interference on the digital information under certain circumstances. In this work the transmission of both In Band - On Channel (AM IBOC) and Digital Radio Mondiale (DRM) digital standards, broadcasting in hybrid mode from adjacent frequencies is analyzed through the use of models, and the implications of the phenomenon and its possible solutions are concluded, taking as example the case of three commercial stations nowadays operating in Mexico City.
\end{abstract}

\section{Keywords}

- digital signals

- $D R M$

- IBOC

- hybrid mode of transmission

\section{Introducción}

Los estándares digitales de radiodifusión In Band-On Channel (IBOC) y Digital Radio Mondiale (DRM) soportan la operación híbrida (transmisión analógica y digital de manera simultánea) de señales de radio en amplitud modulada mediante el envío de las dos señales moduladas, utilizando la técnica de multiplexación por división de frecuencias ortogonales (OFDM) sobre el mismo circuito físico.

La frecuencia central de transmisión asignada a cada estación emisora de ondas analógicas en Amplitud Modulada (cuyo intervalo de frecuencias va de los $535 \mathrm{kHz}$ a los $1605 \mathrm{kHz}$ en México (NOM-01-SCT, 1993), está separada de sus frecuencias adyacentes por medio de una banda de guarda mínima de $10 \mathrm{kHz}$. En la tabla 1 se ilustran el patrón de potencias (horarios diurno y nocturno), utilizado por algunas estaciones de radio.

De acuerdo con los datos mostrados en la tabla 1, la mayor parte de las frecuencias de transmisión están separadas de $30 \mathrm{kHz}$ a $40 \mathrm{kHz}$, pero existen casos en que estas frecuencias están separadas por sólo $20 \mathrm{kHz}$ (tabla 2). Debido a que el modo de transmisión híbrido supone el uso de las frecuencias asignadas a las guardas analógicas para el envío de las señales digitales, esta situación genera problemas de interferencia para algunas frecuencias de transmisión contiguas (la denominada interferencia en banda -Self-in-Band Interference, SII (Chun, 2005), problemas ignorados hasta hoy en las

\begin{tabular}{|c|c|c|c|c|c|c|c|}
\hline \multirow[b]{2}{*}{ Distintivo } & \multirow{2}{*}{$\begin{array}{c}\text { Frecuencia } \\
\text { portadora } \\
{[\mathrm{kHz}]}\end{array}$} & \multicolumn{2}{|c|}{$\begin{array}{c}\text { Potencia de } \\
\text { Transmisión } \\
{[\mathrm{kW}]}\end{array}$} & \multirow[b]{2}{*}{ Distintivo } & \multirow{2}{*}{$\begin{array}{c}\text { Frecuencia } \\
\text { Portadora } \\
{[\mathrm{kHz}]} \\
\end{array}$} & \multicolumn{2}{|c|}{$\begin{array}{c}\text { Potencia de } \\
\text { Transmisión } \\
{[\mathrm{kW}]}\end{array}$} \\
\hline & & Día & Noche & & & Día & Noche \\
\hline $\begin{array}{c}\text { XEDTL } \\
\text { Comunicación }\end{array}$ & 660 & 50 & 1 & $\begin{array}{l}\text { XECMQ El } \\
\text { Fonógrafo }\end{array}$ & 1150 & 20 & 10 \\
\hline XEN La 69 & 690 & 50 & 5 & $\begin{array}{c}\text { XEFR Radio } \\
\text { Felicidad }\end{array}$ & 1180 & 10 & 5 \\
\hline $\begin{array}{l}\text { XEMP Radio } \\
710\end{array}$ & 710 & 10 & 1 & $\begin{array}{c}\text { XEEST } \\
\text { Radio } \\
\text { Noticias } \\
1440\end{array}$ & 1440 & 25 & 1 \\
\hline $\begin{array}{l}\text { XEX Estadio } \\
\text { "W" }\end{array}$ & 730 & 100 & 100 & $\begin{array}{l}\text { XEAI Radio } \\
\text { Fórmula } \\
1470\end{array}$ & 1470 & 50 & 5 \\
\hline
\end{tabular}

Tabla 1. Asignación espectral de algunas estaciones de AM en la ciudad de México (Infraestructura de estaciones de radio AM, 2008) 
mediciones de campo llevadas a cabo, pues las pruebas se han realizado en estaciones "aisladas", sin la comprobación de resultados cuando dos o más estaciones adyacentes con guardas mínimas transmiten al mismo tiempo en modo híbrido.

Es importante mencionar que mientras la interferencia afecta a la señal analógica por los altos niveles de ruido debido al traslape de señales, la señal digital se ve afectada porque su potencia de transmisión es menor que la potencia de la señal analógica, lo que exige, en ambos casos, que la SII se elimine o reduzca antes del proceso de demodulación de la señal.

Tabla 2. Estaciones con guardas de $10 \mathrm{kHz}$ entre ellas

\begin{tabular}{ccc}
\hline $\begin{array}{c}\text { Nombre } \\
\text { de la estación }\end{array}$ & $\begin{array}{c}\text { Identificador de } \\
\text { estación }\end{array}$ & $\begin{array}{c}\text { Frecuencia de } \\
\text { transmisión }(\mathrm{kHz})\end{array}$ \\
\hline XEN La 69 & $\mathrm{r} 1$ & 690 \\
XEMP Radio 710 & $\mathrm{r} 2$ & 710 \\
XEX Estadio “W" & $\mathrm{r} 3$ & 730 \\
\hline
\end{tabular}

Además, aunque la existencia de los problemas de interferencia es común en ambos estándares, el espacio espectral ocupado por sus señales digitales es diferente, lo que condiciona que las soluciones propuestas sean disímiles.

Para limitar los efectos de la interferencia han sido desarrolladas tres propuestas de reducción (y posible eliminación) de la SII: el "método de corte", el "método complementario" y el "método de cancelación $\mathrm{AM}^{\text {" }}$ (Chun, 2005).

Estos métodos se desarrollan brevemente al final del parágrafo "Sistema DRM".

\section{Modulación OFDM}

OFDM es un caso especial de la técnica de multiplexado denominada FDM (Frequency Division Multiplexing), y puede ser considerada técnica tanto de modulación como de canalización que permite transmitir multiportadoras en paralelo (Vázquez, 2009). La canalización en OFDM, se refiere a señales independientes que son subconjuntos de una señal principal separada mediante una modulación apropiada.

Existen dos maneras de implementar las multiportadoras, equivalentes en sus propiedades de transmisión, pero conceptualmente diferentes, la primera utiliza el concepto de multiportadoras como un grupo de filtros adyacentes excitados por un flujo de datos en paralelo, lo que conduce a la transmisión paralela en frecuencia; la segunda se basa en el uso de un grupo de portadoras individuales (Schulze, 2005).
En el primer concepto se consideran slots temporales de longitud fija y una modulación en frecuencia para cada uno; en el segundo, la frecuencia de las subportadoras es fija y la modulación para cada subportadora se realiza en tiempo para cada slot. Para la obtención de los resultados mostrados en el presente documento, se utilizó el segundo concepto.

De esa manera, un flujo de datos con alta tasa de transferencia en serie se divide en subportadoras con bajas tasas de transmisión, cada una de las cuales se modula de manera independiente, lo que equivale a dividir el flujo de datos en K subflujos paralelos, modulados en su propia subportadora a una frecuencia $\mathrm{f}_{k}$ en un dominio complejo, que puede ser descrito por la onda armónica $e^{j 2 \pi f_{k} t}$.

Utilizando los símbolos $s_{k l}$ (donde $k$ representa el número de subportadora y $l$ el bloque de modulación) para la modulación compleja y $g(t)$ para el pulso de transmisión de banda base, es posible ilustrar el proceso OFDM como se muestra en la figura 1.

Mediante la selección de un grupo de frecuencias portadoras ortogonales se obtiene una elevada eficiencia espectral al eliminar la influencia entre subportadoras, traslapando el espectro de cada una de ellas. Una de las consecuencias directas de este arreglo de datos es que el periodo de los símbolos de cada subportadora se vuelve más largo que el retardo presente en el tiempo de transmisión del canal, lo que permite reducir la probabilidad de error en la transmisión.

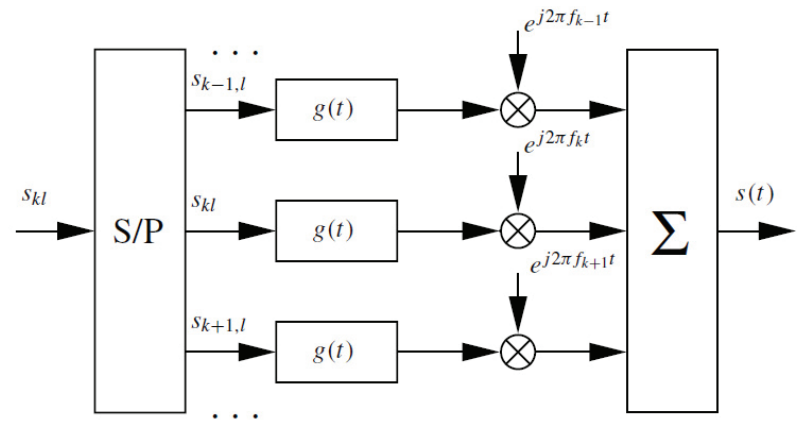

Figura 1. Diagrama de bloques del proceso OFDM (Schulze, 2005)

Una variación de OFDM consiste en la introducción de una codificación de canal a la multiplexación y se denomina COFDM (Coded Orthogonal Frequency Division Multiplexing).

Esta técnica se caracteriza por:

- resistencia al efecto de multitrayectoria (multipath),

- resistencia a pequeños cambios en la distorsión de fase y atenuación de la señal, 
- permiso de uso de redes de frecuencia única,

- posibilitar la transmisión de señal a receptores móviles.

La realización de COFDM consiste en paralelizar un flujo de bits para que, a su vez, sean estos bits los que modulen un conjunto de subportadoras dentro de un canal, conservando el principio de ortogonalidad entre ellas a fin de garantizar la no interferencia. Esta condición posibilita la transmisión sobre un mismo canal de la suma de las subportadoras como una sola señal (Vázquez, 2009) y permite que el receptor descomponga la señal para extraer de ella cada una de las subportadoras, y posteriormente, los bits transmitidos. Al extender los datos a transmitir sobre un gran número de subbandas de frecuencias relativamente cercanas, su reconstrucción se garantiza a partir de la protección de los mismos antes de su transmisión.

Cabe señalar que tanto el espaciamiento entre portadoras como la tasa de bits de cada una de ellas deben satisfacer el principio matemático de ortogonalidad, que indica que cada una de las portadoras puede ser demodulada sin la interferencia de cualesquiera de las otras, a fin de eliminar la existencia de ISI (Inter Symbol Interference).

Para ejemplificar su eficiencia, tomemos un caso particular, supongamos la existencia de un flujo de datos de $1 \mathrm{Mbps}$, que se transmitirán utilizando 500 flujos paralelos, cada uno con una capacidad de $2 \mathrm{kbps}$. Cada uno de estos flujos modularían 500 portadoras, espaciadas cada $2 \mathrm{kHz}$, ocupando un ancho de banda total de $1 \mathrm{MHz}$. Considerando COFDM para el mismo ejemplo, y utilizando una modulación 16-QAM (Quadrature Amplitude Modulation) para cada portadora (que transmite 4 bits por símbolo), deben ser transmitidos únicamente 250000 símbolos por segundo. Cada uno de los 500 flujos paralelos tendría una capacidad de 500 bps, necesitando de $500 \mathrm{~Hz}$ para preservar el principio de ortogonalidad. Así, al ancho de banda requerido se reduce de $1 \mathrm{Mhz}$ a únicamente $2,5 \mathrm{kHz}$, respetando la relación simple entre la tasa de bits, los bits por símbolo, la tasa de símbolos, el espaciamiento entre portadoras y el ancho de banda requerido para el canal (Ahmad et al., 2002). Este arreglo permite transmitir audio con mayor fidelidad sobre bandas frecuenciales usadas actualmente en la transmisión AM.

\section{Sistema AM-IBOC}

Desde comienzos de la década de 1990 se iniciaron los trabajos para el desarrollo de un estándar digital para las estaciones de AM y FM en Estados Unidos, que se resumieron en la recomendación ITU-R BS.774, denominada estándar IBOC (Hoeg et al., 2003).

El estándar, desarrollado por la compañía Ibiquity Digital Corporation, fue aprobado como estándar digital único para AM y FM por la Comisión Federal de Comunicaciones (2002) y adoptado como estándar NRSC-5 por el Comité Nacional de Sistemas de Radio de Estados Unidos (2005). IBOC soporta la transmisión de audio y datos relacionados con la identificación de la melodía o de la emisora, con boletines de tráfico y de clima y otra información útil al escucha, sobre una señal modulada por OFDM.

A fin de realizar una transmisión híbrida, la señal digital deberá transmitirse en bandas laterales ubicadas a cada lado de la señal MF (de frecuencia media) analógica, ocupando un ancho de banda de aproximadamente $10 \mathrm{kHz}$, como se muestra en la figura 2. Como puede distinguirse, existen bandas laterales primarias, secundarias y hasta terciarias (UIT-RBS 1114-6).

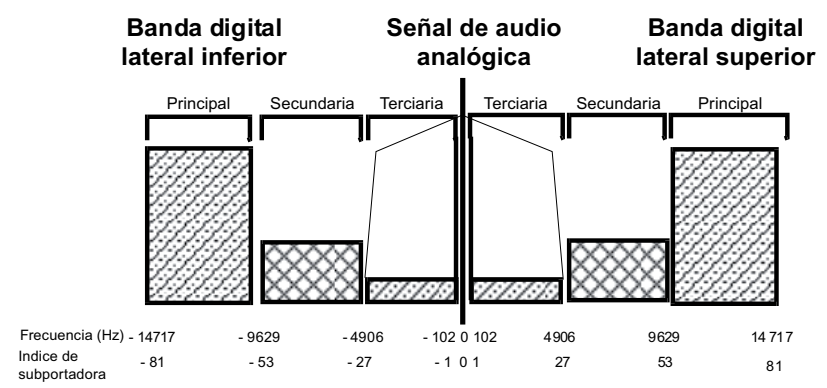

Figura 2. Espectro de la forma de onda híbrida, IBOC (UIT-RBS 1114-6)

La amplitud de las señales analógicas varía en dependencia de la frecuencia central que utilice, así como varía la amplitud de la parte digital de la señal híbrida, pues dentro de cada banda lateral (primaria, secundaria o terciaria) el nivel de cada subportadora se establece con un valor constante, aunque no igual para todas las bandas, debido a que el nivel de potencia se establece con relación directa al nivel de potencia de la señal MF. Así, las bandas superiores primaria y secundaria de la señal r1 (vea la tabla 2) interfiere con las bandas inferiores secundaria y primaria de la señal $\mathrm{r} 2$, respectivamente (figura 3 ).

El escalamiento de las portadoras digitales, establecido en (NRSC-5), se muestra en la tabla 3 (parcialmente). En ella se define que las bandas laterales primarias y secundarias se encuentran, respectivamente, a $30 \mathrm{~dB}$ y $40 \mathrm{~dB}$ por debajo de la potencia de la portadora.

Se hace evidente el problema de interferencia en el sistema AM-IBOC. Las portadoras digitales que no se transmiten en la primera banda adyacente para evitar 


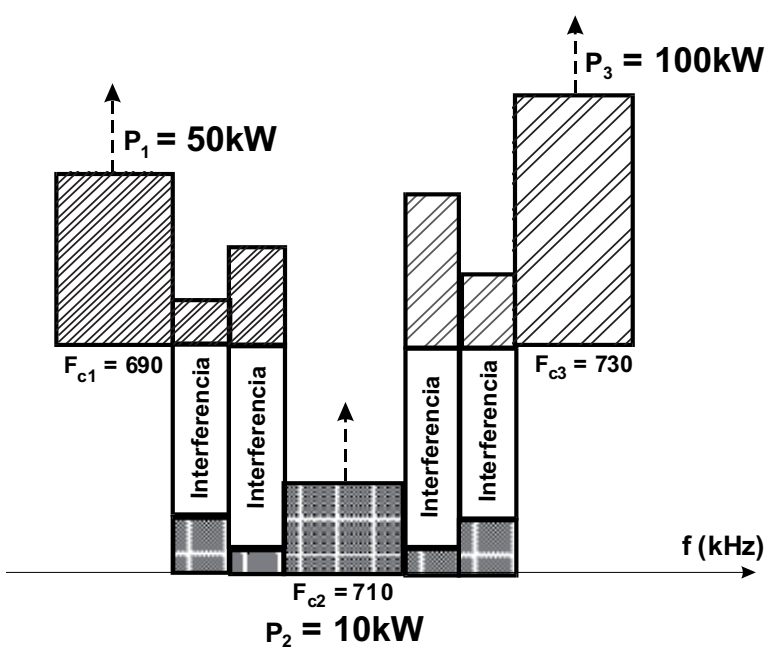

Figura 3. Interferencia de señales digitales entre estaciones adyacentes

Tabla 3. Potencias de portadoras digitales en AM-IBOC modo híbrido

\begin{tabular}{cccc}
\hline $\mathrm{f}_{\text {central }} \mathrm{kHz}$ & $\mathrm{P}_{\text {fcentral }} \mathrm{kW}$ & $\mathrm{P}_{\text {primaria }} \mathrm{kW}$ & $\mathrm{P}_{\text {secundaria }} \mathrm{kW}$ \\
\hline 690 & 50 & 0.05 & 0.005 \\
710 & 10 & 0.01 & 0.001 \\
730 & 100 & 0.1 & 0.01 \\
\hline
\end{tabular}

interferencias, sólo sirven para proteger una pequeñísima parte de las bandas laterales primarias, que son las que forman el núcleo de la señal, pues las bandas secundarias y terciarias no juegan un rol decisivo en la transmisión.

\section{Sistema DRM}

El estándar DRM es un sistema de radiodifusión desarrollado por el consorcio DRM, que se distingue por su balance entre la calidad y la robustez de la señal en los puntos de recepción y por la ocupación de canales con anchos de banda mínimos de $4.5 \mathrm{kHz}$. Utiliza la técnica denominada COFDM, una modulación por amplitud cuadrática QAM16 y mecanismos de corrección de errores en avance con codificación de niveles múltiples (Moumtadi et al., 2009). Estos mecanismos de detección de errores se basan, a su vez, en un código convolucional (Technical Bases for DMR Services Coverage Planning, 2008).

El estándar DRM considera la variación que existe en la propagación de señales en las bandas de AM debidas a deficiencias de aterrizaje físico, al efecto Doppler y al jitter, entre otros factores. Para mejorar los niveles de recepción se diferencian cuatro modos de propagación y, para cada uno de ellos, diferentes modulaciones y tasas de codificación. La elección de una combinación óptima de estos parámetros, combinada con las condiciones de propagación, puede asegurar que la recepción de la señal de audio se apegue a los requerimientos de cobertura y disponibilidad de la estación de radio transmisora.

DRM utiliza un esquema de subportadoras, cuyo número depende del ancho de banda ocupado y del modo de operación, separadas entre sí a la misma distancia para transmitir los datos (DRM. Broad Casters User Manual, 2006). La figura 4 ilustra la situación en el dominio del tiempo y la frecuencia para un esquema con 5 subportadoras adyacentes.

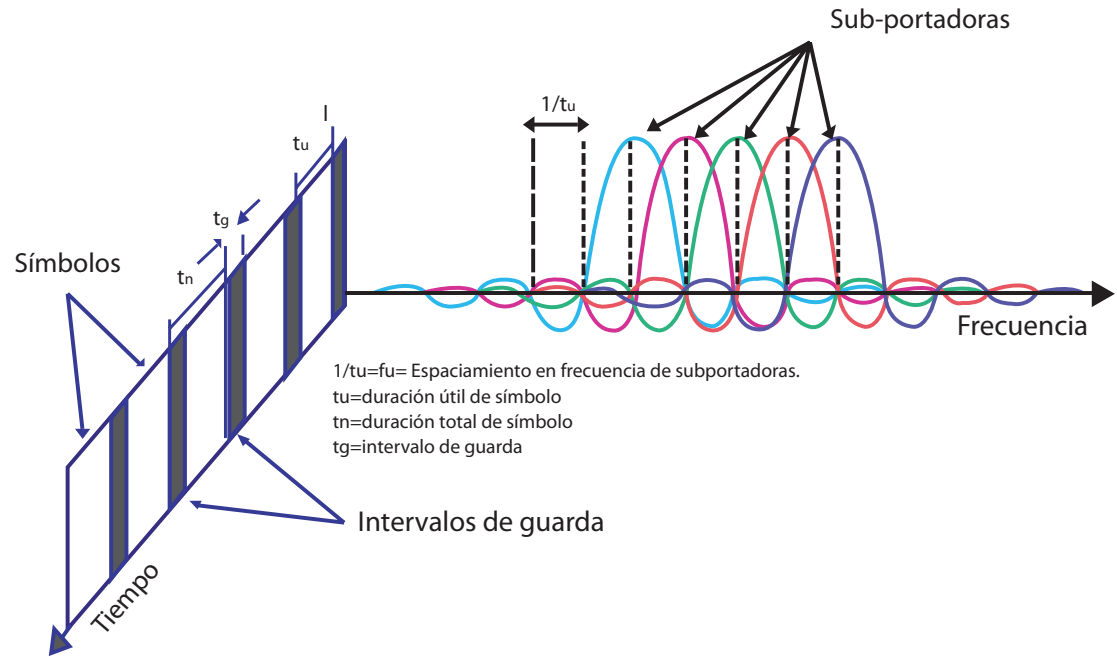

Figura 4. Diagrama de subportadoras DRM en el dominio del tiempo y la frecuencia (Hoeg et al., 2003) 
Como puede observarse en la figura 4, el estándar DRM ocupa únicamente una de las frecuencias de guarda (superior o inferior) de la señal MF para el envío de la señal digital, de manera que puede evitarse la interferencia con los datos de las estaciones adyacentes mediante una apropiada elección de frecuencias de transmisión (figura 5).

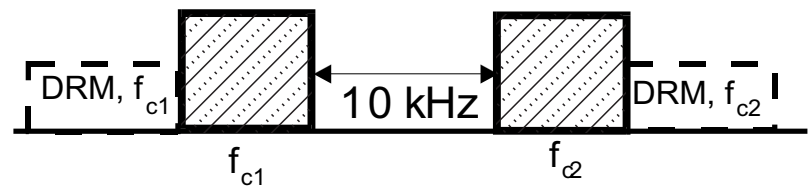

Figura 5. Configuración espectral del sistema DRM con canales de $10 \mathrm{kHz}$ de capacidad

A partir de estas características de formación y transmisión de señal, se han propuesto tres mecanismos de eliminación de IIS, denominados: "método de corte", "método complementario" y "método de cancelación", cuyo funcionamiento puede resumirse como sigue.

El denominado "método de corte", se basa en un filtro que "corta" los tonos con menor potencia de la señal analógica. Este mecanismo permite reducir la interferencia de la señal analógica sobre la digital tanto como se desee: si la calidad del audio de la señal analógica

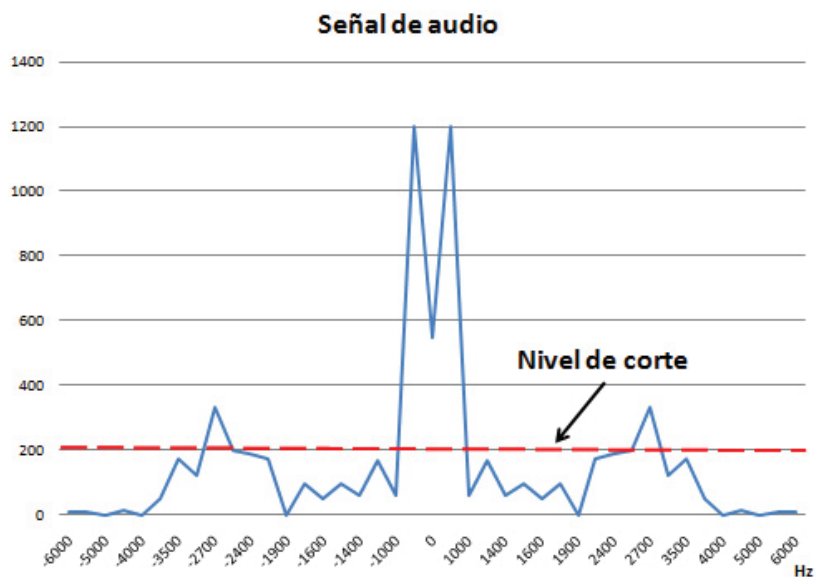

Figura 6. Concepto del "método de corte"

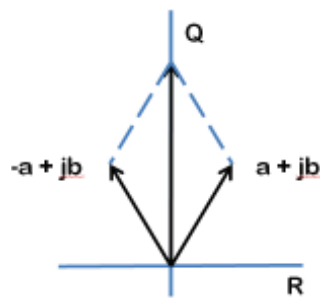

Figura 7. Diseño de señal complementaria depende de la potencia de sus tonos, el problema se reduce a una correcta elección del filtro (figura 6).

Por otro lado, el denominado "método complementario", consiste en el envío de la señal digital en cuadratura con la portadora analógica.

La portadora digital produce un vector que rota respecto a la portadora analógica, afectándola de manera directa. Para evitarlo, se coloca una portadora complementaria en cuadratura respecto a la fase y la frecuencia, asegurando que las señales digitales no se proyecten sobre el eje real, evitando la diafonía de las señales digitales (figura 7).

El tercer mecanismo de eliminación de interferencias, denominado "método de cancelación", se basa en el principio de que las señales de AM son componentes en-fase con una característica simétrica en frecuencia. Si la señal digital se agrega a una sola de las bandas laterales de la señal analógica, su interferencia puede evitarse cancelando la señal desde la banda lateral libre de la señal AM.

Supongamos la existencia de una señal analógica "A" y de su valor simétrico "A*". Entonces, si “ $\mathrm{D}$ " representa la señal digital que se agrega a una sola de las bandas de la señal analógica, es claro que la diferencia entre estas señales simétricas representa el valor de la señal digital (figura 8).

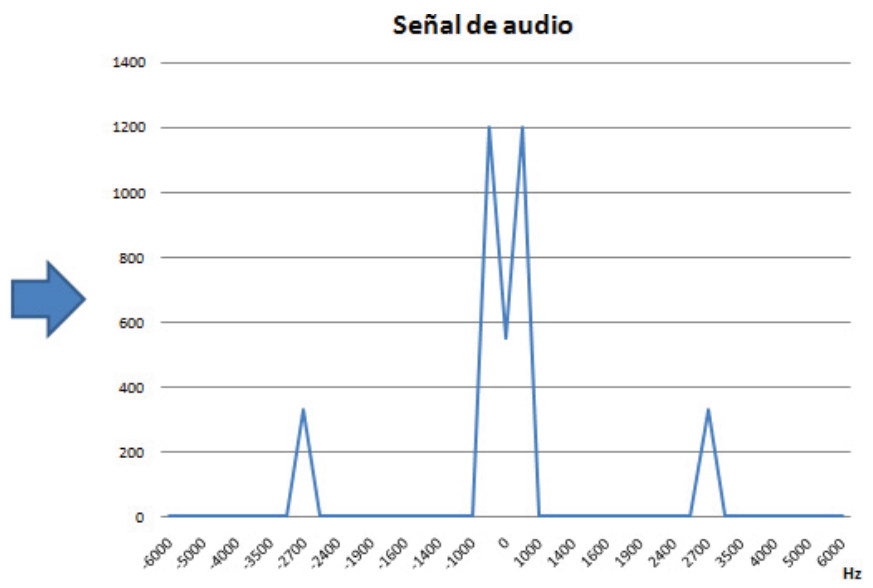

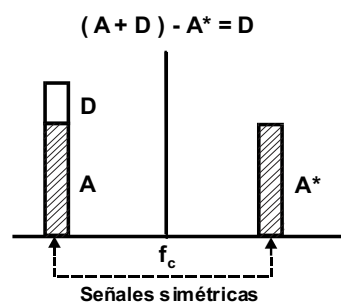

Figura 8. Ejemplificación del "método de cancelación" 
Una vez detalladas las características básicas de las señales IBOC y DRM y de sus mecanismos para evitar la interferencia, pasaremos al desarrollo de los mecanismos de simulación utilizados en el trabajo y su análisis.

\section{Simulación de propagación de señales en AM IBOC}

Para realizar una simulación del comportamiento de propagación de las señales para ambos estándares fue utilizada una computadora equipada con procesador Pentium $^{\mathrm{R}}$ a $2.4 \mathrm{GHz}$, con $756 \mathrm{MB}$ en memoria RAM y, de acuerdo a las recomendaciones de la UIT, a través del software denominado grwave, desarrollado por la UIT, cuyos principios de funcionamiento pueden encontrarse de manera extendida en (UIT-R, P.368-8). Puede apuntarse, sin embargo, que este modelo considera la existencia de una superficie terrestre homogénea limitada por la tropósfera con una variación exponencial de altura. GRWAVE utiliza tres diferentes métodos para evaluar la intensidad del campo: si las distancias son muy largas, se utiliza la denominada serie de residuos y para distancias muy cortas, se utiliza la forma ampliada de la teoría de Sommerfeld de la superficie plana. La óptica geométrica se usa para calcular la intensidad del campo en distancias no cubiertas por los dos métodos anteriores (Vargas, 1993).

Un análisis del código sugiere la existencia de cinco subrutinas:

- módulo de óptica geométrica (GWGO),

- módulo de función de atenuación plana terrestre que utiliza la ecuación de King con la corrección de curvatura de la tierra de Hill y Wait (GWFEC),

- módulo de las series de Hill y Wait para valores mínimos de Q (GWSQ),

- módulo de series residuales (GWRSS),

- módulo de integración numérica (GWINT).

Las gráficas de atenuación que se presentan más adelante se definieron de acuerdo con la relación

$$
E=E_{\text {graf }} \sqrt{\frac{P_{r a d}[k W] D_{0}}{3}}
$$

La cual define que la intensidad de campo eléctrico (E) depende, en su mayor parte, de la raíz cuadrada del producto de la potencia radiada $\left(\mathrm{P}_{\mathrm{rad}}\right)$ por la difracción $\left(\mathrm{D}_{0}\right)$ de la señal. Los gráficos obtenidos muestra un comportamiento linealmente decreciente en regiones cercanas a la antena y exponencialmente decreciente a medida que la onda se aleja de la antena y la difracción asociada a la curvatura de la tierra se vuelve importante (figuras 9 y 10).

Entre los parámetros de entrada más importantes a definir se encuentran la conductividad $(\sigma, \mathrm{S} / \mathrm{m})$, la permitividad relativa del suelo $\left(\varepsilon_{\mathrm{r}}\right)$, la altura de las antenas receptora $\left(\mathrm{h}_{\mathrm{r}}\right)$ y transmisora $\left(\mathrm{h}_{\mathrm{t}}\right)$, así como la frecuencia de operación. Para las gráficas obtenidas se tomó la frecuencia intermedia de cada banda lateral como la representativa de toda esa banda y se utilizaron los valores de entrada mostrados en la tabla 4 . En la columna 4, el valor $\lambda$ representa la longitud de onda correspondiente a la frecuencia en que se efectúa la transmisión. Para la conversión de los valores arrojados por el programa grwave a una potencia de transmisión específica, se considera una directividad de $3.28 \mathrm{~dB}$.

Tabla 4. Valores de entrada para las gráficas de simulación

\begin{tabular}{|cccc|}
\hline$\sigma(\mathrm{S} / \mathrm{m})$ & $\varepsilon_{\mathrm{r}}$ & $\mathrm{h}_{\mathrm{r}}(\mathrm{m})$ & $\mathrm{h}_{\mathrm{t}}(\mathrm{m})$ \\
\hline 50 & 80 & 2 & $\lambda / 2$ \\
\hline
\end{tabular}

Los valores de conductividad y permeabilidad relativa del suelo son los correspondientes a los de agua fresca, condicionados por la construcción de la Ciudad de México sobre un lago. Una mala elección de estos parámetros generaría importantes errores en el cálculo de intensidad del campo de las señales, con respecto a los valores que se han obtenido en las pruebas de campo realizadas (ITU, 2005).

En la figura 9 se muestra la comparación de los valores de la atenuación del campo eléctrico para dos señales adyacentes: la señal $\mathrm{r} 1\left(f_{c}=690 \mathrm{kHz}\right)$ con una antena de 217 metros de altura (con valores en sus bandas digitales superior secundaria (Upper Secondary, US) de $\mathrm{f}_{\mathrm{US}}=698 \mathrm{kHz}$ y superior primaria (Upper Primary, UP) de $\mathrm{f}_{\text {UP }}=703 \mathrm{kHz}$ y la señal $\mathrm{r} 2\left(\mathrm{f}_{c}=710 \mathrm{kHz}\right)$ con una antena de $211 \mathrm{~m}$ de altura y valores de bandas laterales inferior secundaria (Lower Secondary, LS) de $\mathrm{f}_{\mathrm{LS}}=703$ $\mathrm{kHz}$ e inferior primaria (Lower Primary, $L P$ ) de $\mathrm{f}_{\mathrm{LP}}=698$ $\mathrm{kHz}$. Puede observarse que la banda lateral secundaria superior de r1 interfiere con la banda lateral primaria inferior de r2 únicamente a casi 1000 kilómetros de distancia de la antena (aunque la intensidad del campo eléctrico ya es muy baja). Por otro lado, las señales de la banda lateral primaria superior de la señal r1 y la señal de la banda lateral inferior secundaria de r2 son casi indistinguibles a cualquier distancia y prácticamente idénticas a 100 kilómetros de la antena.

En la figura 10 se muestra la atenuación del campo eléctrico para la banda superior de la señal r2. Se puede observar que el comportamiento es muy parecido al de la figura 9, pues la frecuencia varía muy poco. 

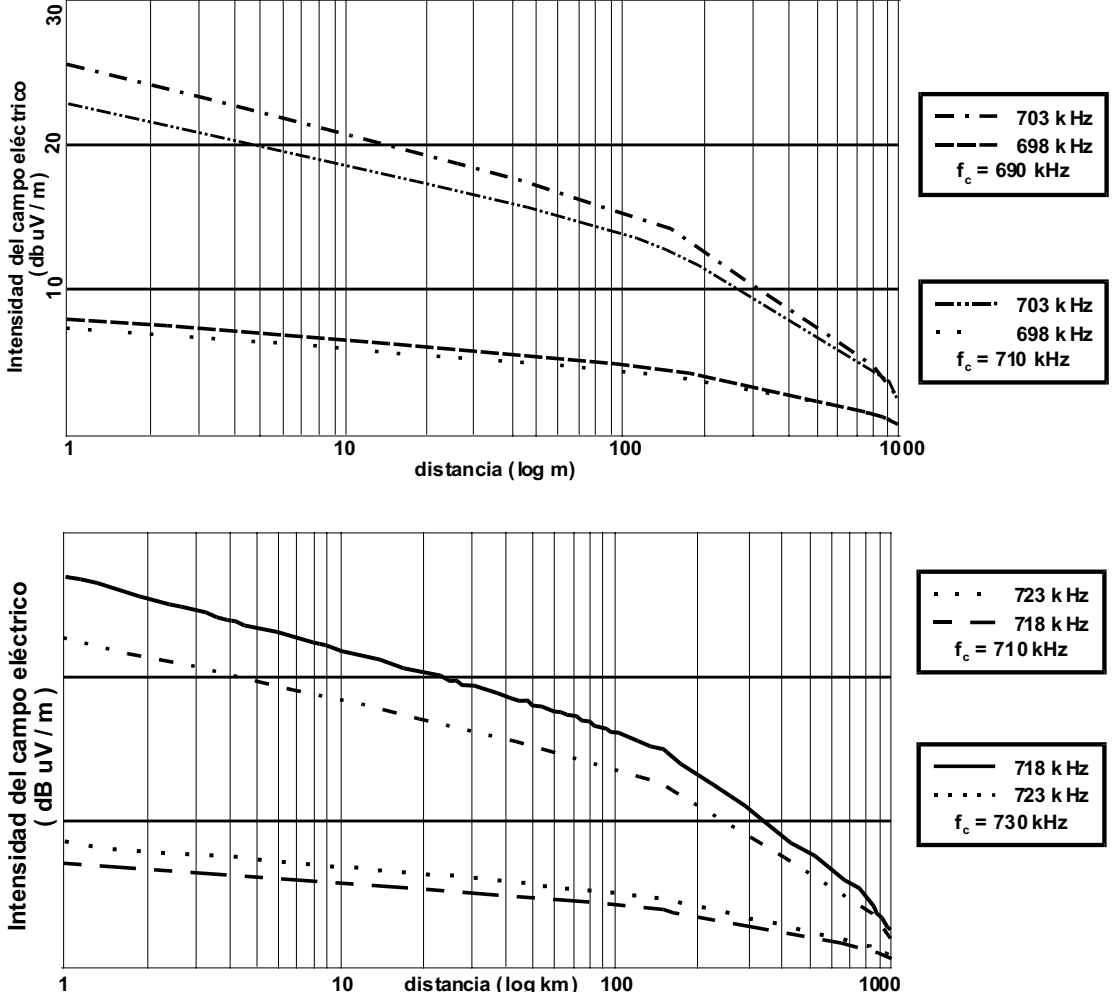

Figura 9. Atenuación del campo eléctrico con respecto a la distancia para $\mathrm{f}_{\mathrm{c} 1}=690$ (superiores) y para $\mathrm{f}_{\mathrm{c} 2}=710$ (inferiores)
Figura 10. Atenuación del campo eléctrico con respecto a la distancia para $\mathrm{f}_{\mathrm{c} 2}=710$ (superiores) y para $\mathrm{f}_{\mathrm{c} 3}=730$ (inferiores)
Para la señal r3 se modeló una antena transmisora con una altura de $205 \mathrm{~m}$. En la figura 9 se mostró la atenuación del campo eléctrico de las bandas inferiores, que genera interferencia con las bandas laterales superiores de la señal r2 (figura 10). Un primer análisis de la interferencia entre estaciones en el sistema AM IBOC permite afirmar, a partir de las gráficas ( 9 a 12), que si dos estaciones transmiten al mismo tiempo y desde frecuencias adyacentes con guardas mínimas de separación, es imposible evitar la interferencia entre ellas.

\section{Simulación de propagación de señales en DRM}

Para llevar a cabo la simulación de señales en el estándar DRM se asignaron los mismos valores de entrada que en IBOC, a fin de posibilitar la comparación de los resultados obtenidos, variando sólo los datos relativos a la potencia de transmisión, como característica propia del estándar.

La figura 11 muestra la atenuación sufrida por el campo eléctrico de la señal r1. Se tomó la frecuencia intermedia $(680 \mathrm{kHz})$ como representativa del bloque que comprende la parte de la señal digital de DRM.

En la figura 12 se reproducen los valores obtenidos de la atenuación que sufre el campo eléctrico de la señal r2 con respecto a la distancia recorrida.

Una tercera gráfica muestra los resultados obtenidos para la parte digital de la señal r3 (figura 13).

\section{Áreas de cobertura y análisis de resultados}

A partir de las gráficas (11 a 13) puede realizarse un diagrama de cobertura de las estaciones y observar cómo podrían interferir una con otra, dependiendo de su ubicación geográfica.

Es posible formular a priori la hipótesis de que, en DRM, las estaciones pueden tener buena área de cobertura sin que la interferencia de otras señales les afecte de manera sustancial, gracias a la capacidad de selectividad que poseen los equipos receptores y el umbral de recepción especificado en (UIT-R, BS.703) para la banda de frecuencias $60 \mathrm{~dB} \mu \mathrm{V} / \mathrm{m}$ (o hasta 54 y $40 \mathrm{~dB} \mu \mathrm{V} / \mathrm{m}$, según la misma referencia) para obtener una relación entre señal deseada y señal no deseada de $26 \mathrm{~dB}$ en los limites protegidos.

Sin embargo, la medición de esta relación, que es necesario regular en DRM, arroja resultados de hasta 15-20 dB menores a la relación de las señales analógicas, conservando niveles de calidad de audio más altas y con áreas de cobertura comparable (ITU, 2005).

En el estándar IBOC el comportamiento de la señal se complica, pues existe el traslape de las señales en el dominio de la frecuencia debido a que IBOC utiliza dos señales del espectro, aunque sean parte de la misma emisión. 

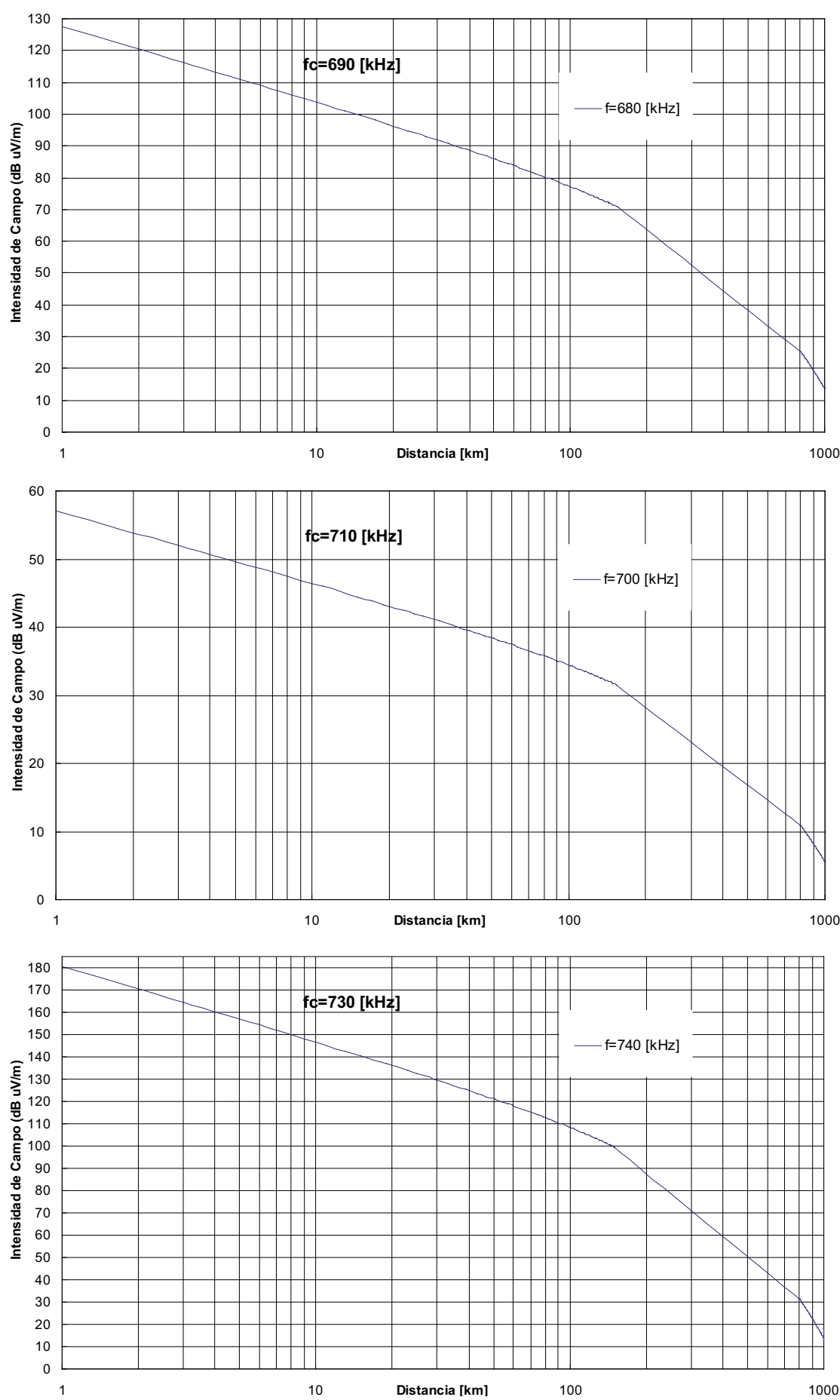

Figura 11. Atenuación del campo eléctrico de la parte digital de una señal DRM para $\mathrm{f}_{\mathrm{c} 1}=690 \mathrm{kHz}$

Figura 12. Atenuación del campo eléctrico de la parte digital de una señal DRM, $f_{c 2}=710 \mathrm{kHz}$

Figura 13. Atenuación del campo eléctrico de la señal DRM para $\mathrm{f}_{\mathrm{c} 3}=730 \mathrm{kHz}$
La UIT ha determinado que si una señal analógica se reemplaza por una digital (DRM o AM IBOC), la potencia de transmisión promedio de la señal digital debe ser reducida entre 6 y $7 \mathrm{~dB}$ para proveer la misma protección entre estaciones (UIT-R, BS 1514-1); dicho de otra manera, dada una transmisión digital con una potencia determinada, se causará entre 6 y 7 dB más interferen- cia a la salida de un receptor digital, que a una señal AM con la misma potencia.

Para el cálculo de la interferencia de señales, se simula la transmisión desde tres estaciones de radio actualmente en explotación en la Ciudad de México (figura 14), correspondientes a las referenciadas en la tabla 2. En la figura se marcan además las distancias 


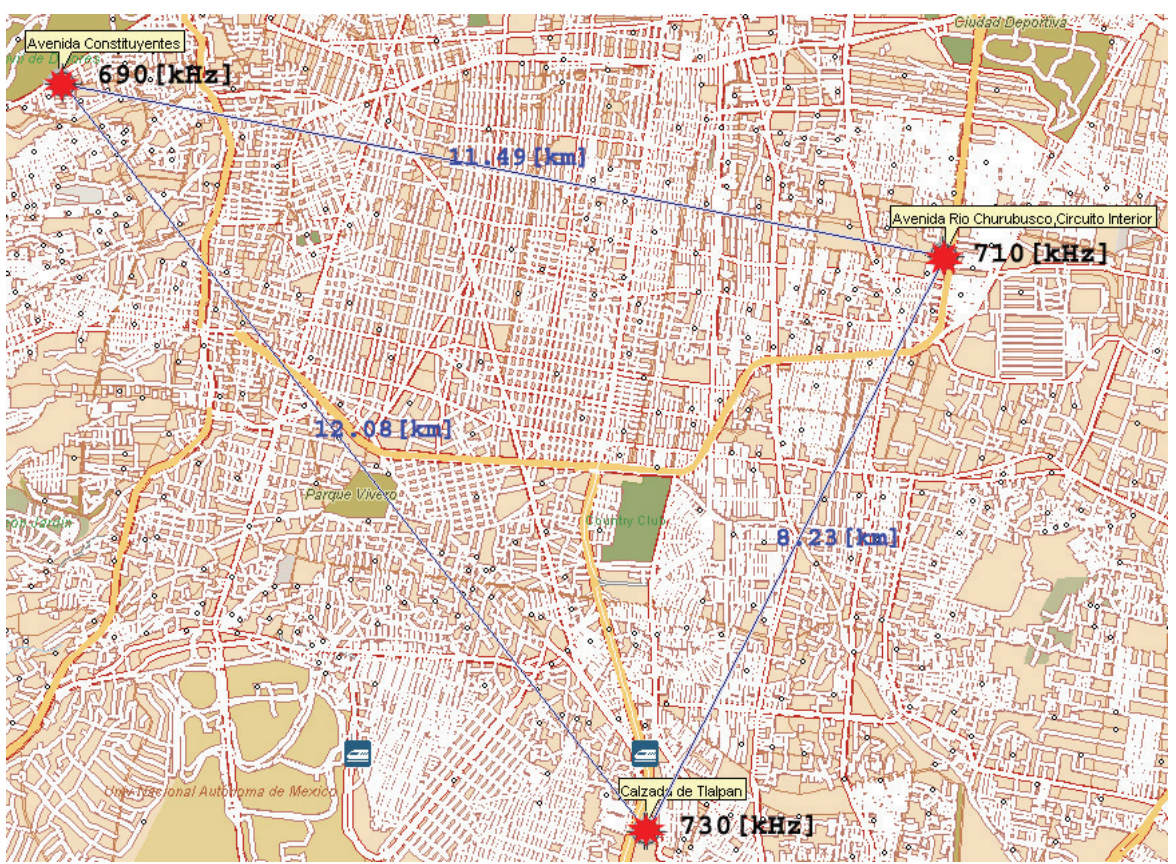

Figura 14. Ubicación de las estaciones de transmisión bajo estudio entre ellas y las avenidas principales cercanas a las estaciones.

La distancia entre estaciones transmisoras se resume en la tabla 5.

Al calcular las distancias de cobertura de señal bajo el estándar AM IBOC para cada una de las tres estaciones en estudio, considerando un umbral de $60 \mathrm{~dB} \mu \mathrm{V} / \mathrm{m}$ para las estaciones con mayor potencia de transmisión, se obtienen los resultados mostrados en la tabla 6 .

Tabla 5. Distancia entre estaciones de transmisión

\begin{tabular}{cc}
\hline Pares de estaciones & Distancia, kms \\
\hline $690 \mathrm{kHz}$ a $710 \mathrm{kHz}$ & 11.49 \\
$710 \mathrm{kHz}$ a $730 \mathrm{kHz}$ & 8.23 \\
$730 \mathrm{kHz}$ a $690 \mathrm{kHz}$ & 12.08 \\
\hline
\end{tabular}

Tabla 6. Cobertura máxima aproximada del estándar AM IBOC

\begin{tabular}{ccc}
\hline Estaciones de referencia & $\begin{array}{c}\text { Identificador de } \\
\text { estación }\end{array}$ & Cobertura $(\mathrm{km})$ \\
\hline $690 \mathrm{kHz}$ & $\mathrm{r} 1$ & 220 \\
$710 \mathrm{kHz}$ & $\mathrm{r} 2$ & 39 \\
$730 \mathrm{kHz}$ & $\mathrm{r} 3$ & 400 \\
\hline
\end{tabular}

Se puede apreciar que el área de cobertura de las estaciones es mucho mayor que la distancia existente entre ellas, lo cual implica que existen áreas de cobertura común en las que puede presentarse interferencia en las señales.
El comportamiento de las señales r 1 y r2 en las áreas de cobertura común tendrá un comportamiento como el que se ilustra en la figura 15.

En la figura 15 el punto de referencia es el de la estación con menor potencia (r2), por lo que el pico de potencia máxima de la señal $\mathrm{r} 1$ aparece a una distancia de $11.5 \mathrm{~km}$ aproximadamente (tabla 4). La línea marcada con triángulos, denominada "diferencia" muestra la relación de potencias entre ambas señales, pudiéndose observar que el valor siempre es mayor a los $20 \mathrm{~dB}$ $(\mu \mathrm{V} / \mathrm{m})$ y siempre a favor de la señal $\mathrm{r} 2$. Por esta razón, cualquier receptor ubicado dentro del radio de cobertura de r1 está sujeto a captar la señal de r2 también, y dado que la potencia de esta última es siempre mayor, la señal r1 será desechada, y el receptor seleccionará la señal de r2 como la adecuada para procesar. En términos estrictos, la señal digital de las bandas inferiores a la emisora $\mathrm{r} 2$ serán reconocidas por los receptores como ruido sujeto a filtrado.

El siguiente caso es muy parecido al anterior, en esta ocasión las bandas superiores digitales de la señal r2 interfieren con las bandas digitales inferiores de la señal r3, como se muestras en la figura 16.

Nuevamente, el punto de referencia distancia 0 se tomó como la ubicación de la estación transmisora r2, por eso el pico de máxima potencia de transmisión aparece en aproximadamente $9 \mathrm{~km}$ para $\mathrm{r} 3$. El radio de cobertura máximo de r1 es de $39 \mathrm{~km}$, que en este caso corresponden a las portadoras digitales de la banda superior. Sin embargo, estas señales interfieren con las ban- 

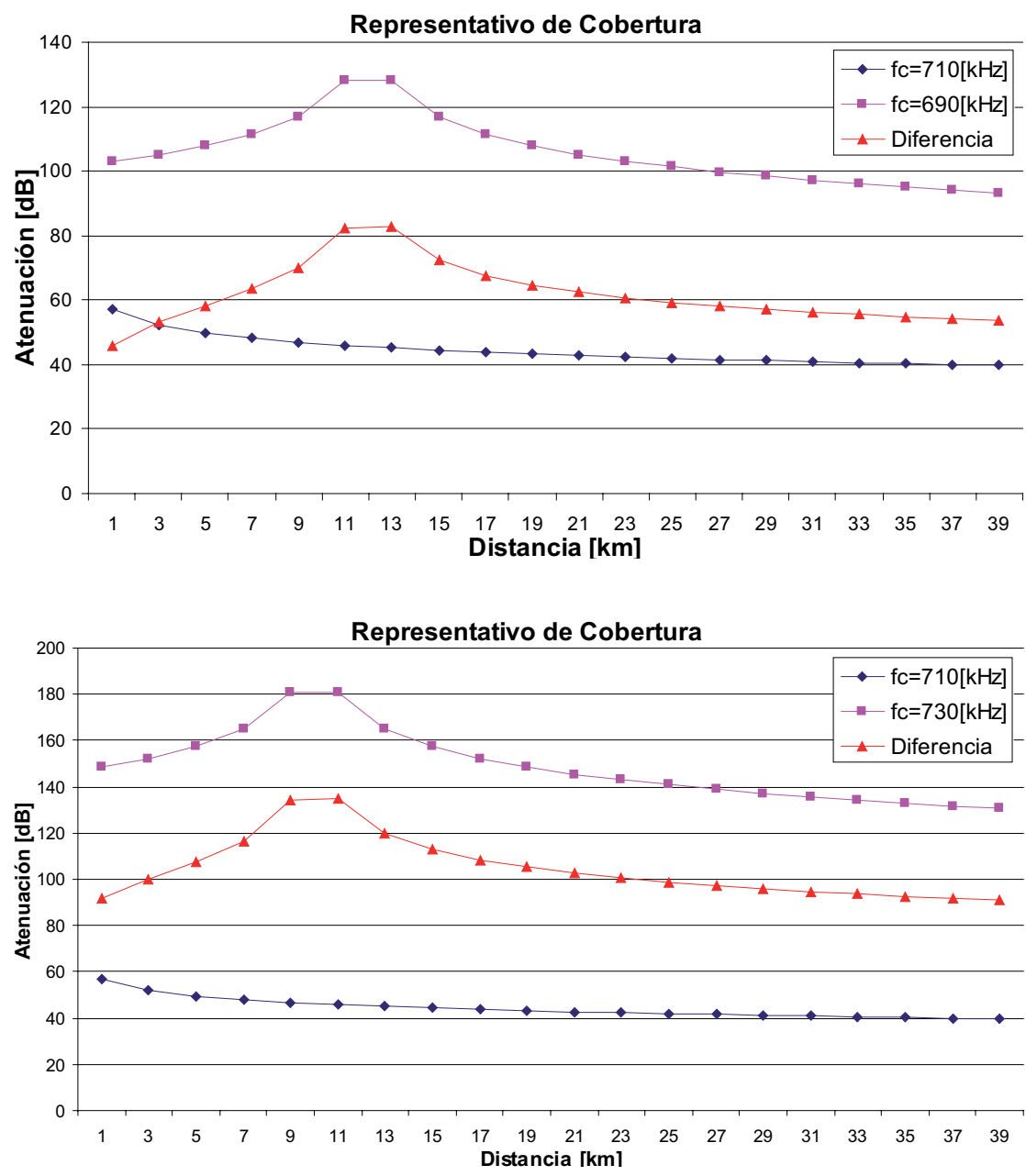

Figura 15. Cobertura de señales e interferencia entre ellas, estándar AM IBOC
Figura 16. Cobertura de señales e interferencia entre ellas, estándar AM IBOC das laterales digitales inferiores de $r 3$, que tienen una potencia de transmisión mucho mayor a la de las bandas laterales digitales superiores de r2. La diferencia entre las potencias de ambas bandas es más pronunciada que en el primer caso, pero el resultado es el mismo: un receptor cualquiera ubicado el área de cobertura común desechará la señal r2, por considerarla ruido.

En cuanto al sistema DRM, la flexibilidad del estándar permite anular las interferencias entre las emisoras bajo estudio, debido a que el segmento espectral utilizado para las componentes digitales de cada emisora es distinto (figura 5), aun en el caso de que las áreas de cobertura coincidieran.

\section{Conclusiones}

En este trabajo se expusieron los posibles problemas de interferencia que pueden surgir al adoptar algún sistema de radiodifusión sonora digital en la banda de ondas media en operación diurna.
Los resultados obtenidos del análisis de ocupación espectral, así como los resultados obtenidos de las simulaciones, muestran claramente las ventajas y desventajas de los estándares que operan en esta banda (DRM y AM IBOC).

Ambos sistemas ofrecen la ventaja de poder utilizar las bandas actualmente asignadas a la radio AM, evitando utilizar un nuevo intervalo espectral y realizar un cambio fundamental de la infraestructura física de las estaciones de radiodifusión. Además, la potencia necesaria para la transmisión de las señales digitales es mucho menor que la que actualmente se requiere para señales analógicas, lo que repercute en el consumo de energía de las emisoras, hecho que aunado a la expansión de servicios ofrecidos en la misma transmisión y a la liberación de la banda de guarda de su función de simple espacio espectral entre señales útiles, abre la puerta a nuevos concesionarios para entrar al mercado de la radio digital, ofreciendo al usuario una mayor base de elección de servicios. 
Cabe hacer la aclaración, sin embargo, que esta ventaja técnica se ve limitada por las condiciones jurídicas y comerciales de explotación de los estándares. En efecto, el sistema IBOC no es un sistema abierto y cualquier emisora que desee utilizarlo debe pagar cierta tarifa por el uso de la tecnología además de la adquisición del equipo necesario. En contraste, DRM sólo requiere que los concesionarios compren el equipo para la generación de la señal, sin cobro de tarifa alguna.

Entre las desventajas que se concluyen como resultado de la simulación se tiene que el estándar AM IBOC presenta requerimientos espectrales que imposibilitan la transmisión desde estaciones cuyas frecuencias centrales de operación están separadas por guardas mínimas.

Durante el periodo de transmisiones en modo híbrido, esto equivale a que una estación quede sin posibilidades de transmitir eficientemente señales digitales, esto es, servicios agregados, con la consecuente desventaja comercial.

El estándar DRM, gracias a su flexibilidad de ocupación espectral, permite trabajar con anchos de banda muy reducidos. La gama de servicios agregados proporcionados durante el período de transición de señales es limitada, pero proporciona en cambio, una calidad de audio insuperable al evitar la interferencia de señales.

\section{Referencias}

Ahmad R, Burton R. Multi-Carrier Digital Communications: Theory and Applications of FDMA, Kluwer Academic Publishers, Information Technology: Transmission, Processing and Storage Series, USA, 2002, 216 pp.

Chun-Jen F. Signals Design of Tertiary Sideband on Hybrid IBOC AM Systems, Institute of Computer and Communication Engineering, National Chen Kung University, Taiwan, 2005.

DRM Broadcasters' User Manual, 2006 [en línea]. Disponible en: http://www.drm.org/fileadmin/media/downloads/Broadcast_Manual.pdf
Hoeg W., Lauterbach T. Digital Audio Broadcasting: Principles and Applications of Digital Radio, 2a ed., UK, John Wiley and Sons, 2003.

Infraestructura de Estaciones de Radio AM [en línea]. Disponible en: http://cofetel.gob.mx/wb/Cofetel_2008/Cofe_estaciones_ de_am_in

ITU. Digital Radio Mondiale DRM Local Coverage Using the 26 mhz Broadcasting Band, 2005 [en línea]. Disponible en: http:// www.drm.org/fileadmin/media/downloads/THE_26-MHz BROADCASTING_BAND.pdf

Moumtadi, Delgado, Cruz. Desarrollo de un software de simulación de patrones de recepción para señales móviles DRM en la banda de los 26MHz. Memorias del Congreso COPIOS 2009, Perú.

NRSC-5. In-Band / On-Channel digital Radio Broadcasting Standard.

Schulze L. Theory and Applications of OFDM and CDMA-Wideband Wireless Communications, John Wyley and Sons, UK, 2005, 408 pp.

SCT (Secretaría de Comunicaciones y Transportes), 1993 Norma Oficial Mexicana NOM-01-SCT1-93.

Technical Bases for DRM Services Coverage Planning [en línea]. Disponible en: http://www.drm.org/fileadmin/media/downloads/June_2008_EBU_Technical_Bases_for_DRMservices_ coverage_planning.pdf

UIT-R, BS 1114-6 Recomendation. Sistemas de radiodifusión sonora digital terrenal para receptores en vehículos, portátiles y fijos en la gama de frecuencias $30-3000 \mathrm{MHz}$.

UIT-R, P.368-8 Recomendation. Ground-Wave Propagation Curves for Frequencies Between $10 \mathrm{kHz}$ and $30 \mathrm{MHz}$.

UIT-R, BS.703 Recommendation. Characteristics of AM Sound Broadcasting Reference Receivers for Planning Purposes.

UIT-R, BS.1514-1 Recommendation. Sistema para radiodifusión sonora digital en las bandas de radiodifusión por debajo de 30 $\mathrm{MHz}$, Anexo 4.

Vargas-Dávila C. Comparison of HF Groundwave Propagation Models, Naval Postgraduate School, Monterey, California, 1993.

Vázquez-Reyes J.A. Estudio comparativo técnico-económico de los estándares terrestres de radiodifusión digital DAB e IBOC, Facultad de Ingeniería, Universidad Nacional Autónoma de México, junio de 2009. 


\section{Semblanza de los autores}

Fatima Moumtadi. Obtuvo su maestría en sistemas de radiodifusión satelital y su doctorado en televisión por la Facultad de Radiodifusión y Televisión de la Universidad Técnica de Comunicaciones e Informática de Moscú, Rusia (MTUCI). Se desarrolló profesionalmente en el área de radiofrecuencia. Ha publicado artículos en congresos y revistas nacionales e internacionales. Actualmente es profesora de carrera en el Departamento de Electrónica en la Facultad de Ingeniería de la Universidad Nacional Autónoma de México.

Julio Carlos Delgado-Hernández. Culminó cursos de especialización en sistemas de conmutación y de transmisión digital (1996) y de doctorado en ciencias técnicas (2002) en la Facultad de Sistemas de Electrocomunicación de la Universidad Técnica de Comunicaciones e Informática de Moscú, Rusia (MTUCI). Durante 4 años, hasta junio de 2009, se desempeñó como director de Networking de la empresa Interstice net-@SA de CV, actividad que combinó con labores de docencia en la Universidad del Valle de México, en la Universidad Anáhuac del Norte y en la Escuela Militar de Ingenieros. Desde enero de 2010, funge como director general de la empresa TINNPRO S de RL de CV.

Javier Rodríguez-Huerta. Egresó de la carrera de ingeniero en telecomunicaciones del Departamento de Telecomunicaciones de la Facultad de Ingeniería de la Universidad Nacional Autónoma de México en 2009. 